\section{TECHNOLOGY}

\section{It's a knock-down}

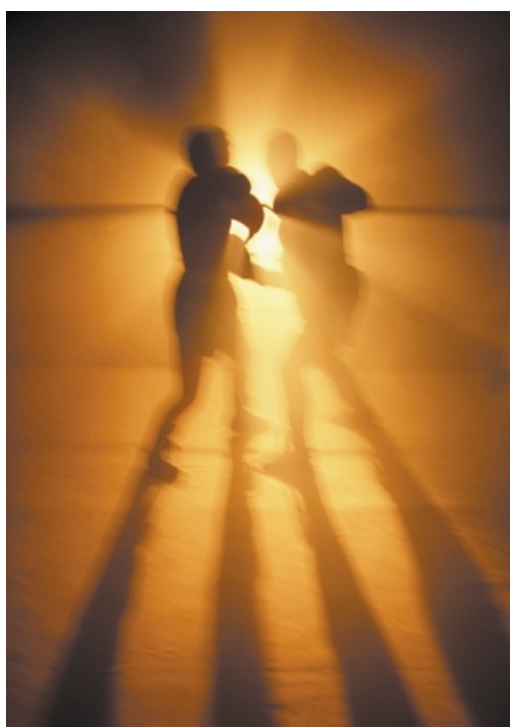

Since its discovery in Caenorhabditis elegans, RNA interference (RNAi) has revolutionized gene-function analysis. The success of RNAi lies in its simplicity - when introduced into cells of certain organisms, doublestranded (ds) RNAs can cause a sequence-specific mRNA degradation that mimics a loss-of-function phenotype. Elbashir and colleagues now show for the first time that RNAi can work consistently and specifically in mammalian cells in response to short dsRNA molecules (called siRNAs). Their study also reveals the existence of two pathways - one general and one sequence specific that are activated in mammalian cells in response to dsRNA.
For those interested in gene function, RNAi-mediated gene knock-down is a useful and rapid alternative to gene knockouts or conventional antisense technology, and it works efficiently in plants and in many invertebrates. Although RNAi has previously been shown to work in mammalian oocytes and embryos, in mammalian cells dsRNAs longer than 30 nucleotides cause a general, sequence non-specific, mRNA degradation and translational shutdown. As very short dsRNAs mediate specific RNAi effects in Drosophila, Elbashir et al. tested whether such siRNAs might also work in mammalian cells. They synthesized 21-mer dsRNA molecules with sequence homology to three luciferase genes and co-transfected plasmids containing these genes with the corresponding siRNAs into four different types of mammalian cell lines. The authors determined siRNA effectiveness by measuring luciferase luminescence and found that, although the extent of suppression varies - possibly depending on the level of target gene expression - suppression is completely sequence specific. Furthermore, siRNA is effective at significantly lower concentrations than antisense or ribozymes in gene-targeting experiments. Similar results were also obtained for suppressing endogenous genes.

Elbashir et al. investigated the effects of longer, supposedly nonspecific, dsRNAs and found that they also act in a sequence-specific manner, but that this is normally masked by the non-specific effects. Their observations indicate that there are two pathways in mammalian cells that can be activated in response to dsRNAs.

RNAi provides unequalled flexibility for rapidly analysing gene function and can be used to

\title{
Opening the flood gates?
}

It's been a long time coming, but now two papers report a clear-cut identification by linkage mapping of a gene involved in a common human disorder - Crohn disease (CD). Importantly, they also indicate how the innate immune system might be involved in the aetiology of $\mathrm{CD}$, because the identified gene - NOD2 - encodes an intracellular receptor for bacterial lipopolysaccharides (LPS) that activates NFKB, a target of the innate immune signalling pathway and a transcriptional regulator of inflammatory genes. $\mathrm{CD}$ is a chronic inflammatory gut disorder, thought to be caused by an abnormal inflammatory response to enteric microbes. In 1996, a CD susceptibility locus, $I B D 1$, was identified on chromosome 16. Little progress has been made since, but it is this locus that the two research teams one European, the other US-based tackled in their studies, using positionalcloning and candidate-gene strategies, respectively.
Hugot $e$ al. took a decisive step when they identified association of $\mathrm{CD}$ to an allele of a chromosome-16 microsatellite marker. Despite the borderline significance of this association, the authors went on to identify putative transcripts in the region of this marker, and identified over 30 single nucleotide polymorphisms (SNPs) by sequencing the region from affected and unaffected individuals. Several turned out to be non-synonymous variants in a chromosome-16 gene, NOD2. Three of these SNPs - each independently associated with disease susceptibility - altered the leucinerich repeat (LRR) region of NOD2, which is required for $L P S$ recognition.

Having previously identified NOD2, Ogura et al. considered it a candidate for $\mathrm{CD}$ because of its chromosome-16 location. On sequencing the gene from $\mathrm{CD}$ individuals, they identified an insertion that caused two frameshift mutations in the LRR region and the premature truncation of NOD2. In in vitro assays, this mutant NOD2 produced considerably diminished levels of NFKB activation in response to bacterial LPS compared to wild-type NOD2.
So how could NOD2 contribute to susceptibility to $\mathrm{CD}$ ? The innate immune system regulates the immediate immune response to bacterial pathogens, components of which are recognized in host immune cells by specific receptors, such as NOD2. A defect in this recognition might lead to an exaggerated inflammatory reaction being mediated by the adaptive immune system. Alternatively, NOD2 might act to trigger cytokines that dampen inflammatory responses. Although NOD2 does not account for all susceptibility to $\mathrm{CD}$, it does provide a first glimpse into the aetiology of the disease and should speed the discovery of other CD loci and future therapies, and improve its diagnosis. As John Todd comments in an accompanying News and Views, these papers are hopefully the first of many such successes in grappling with the genetic basis of multifactorial, common disease.

(1) References and links ORIGINAL RESEARCH PAPERS Hugot, J.-P. et al. Association of NOD2 leucine-rich repeat variants with susceptibility to Crohn's disease. Nature 411, 599-603 (2001) | Ogura, Y. et al. A frameshift mutation in NOD2 associated with susceptibility to Crohn's disease. Nature 411, 603-606 (2001)

FURTHER READING Todd, J. A. Tackling common disease. Nature 411, 537-539 (2001) WEB SITE NIH Crohn disease web links 
determine gene function on a genome-wide scale, as recently shown in C. elegans. The findings of Elbashir et al. have important consequences for those investigating gene function in mammalian cells. But before these applications become practical in mammalian systems, it will be essential to establish easy and efficient ways of delivering siRNA into mammalian cells and rapid phenotypic screens. Questions regarding the biological role of endogenous dsRNAs in gene regulation also remain to be answered.

Magdalena Skipper

(20) References and links ORIGINAL RESEARCH PAPER Elbashir, S. M. et al. Duplexes of 21-nucleotide RNAs mediate RNA interference in cultured mammalian cells. Nature 411, 494-498 (2001)

FURTHER READING Bass, $B$. The short answer. Nature 411, 428-429 (2001) | Hammond, S. M. et al. Post-transcriptional gene silencing by double-stranded RNA. Nature Rev. Genet. 2, 110-119 (2001)

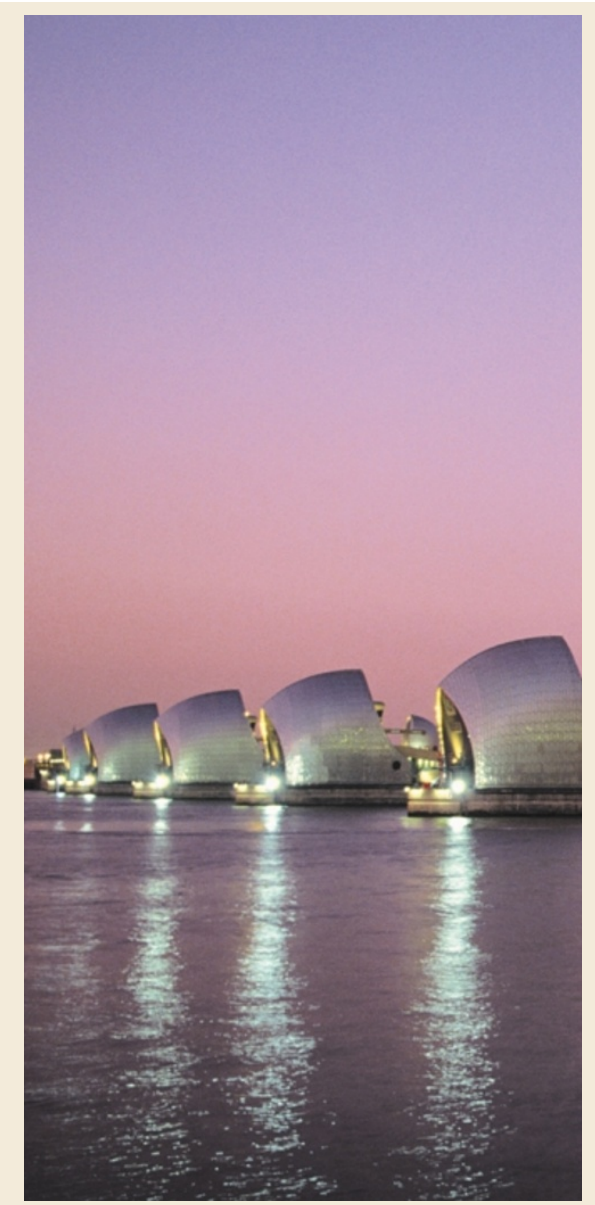

The Thames flood barrier, UK.

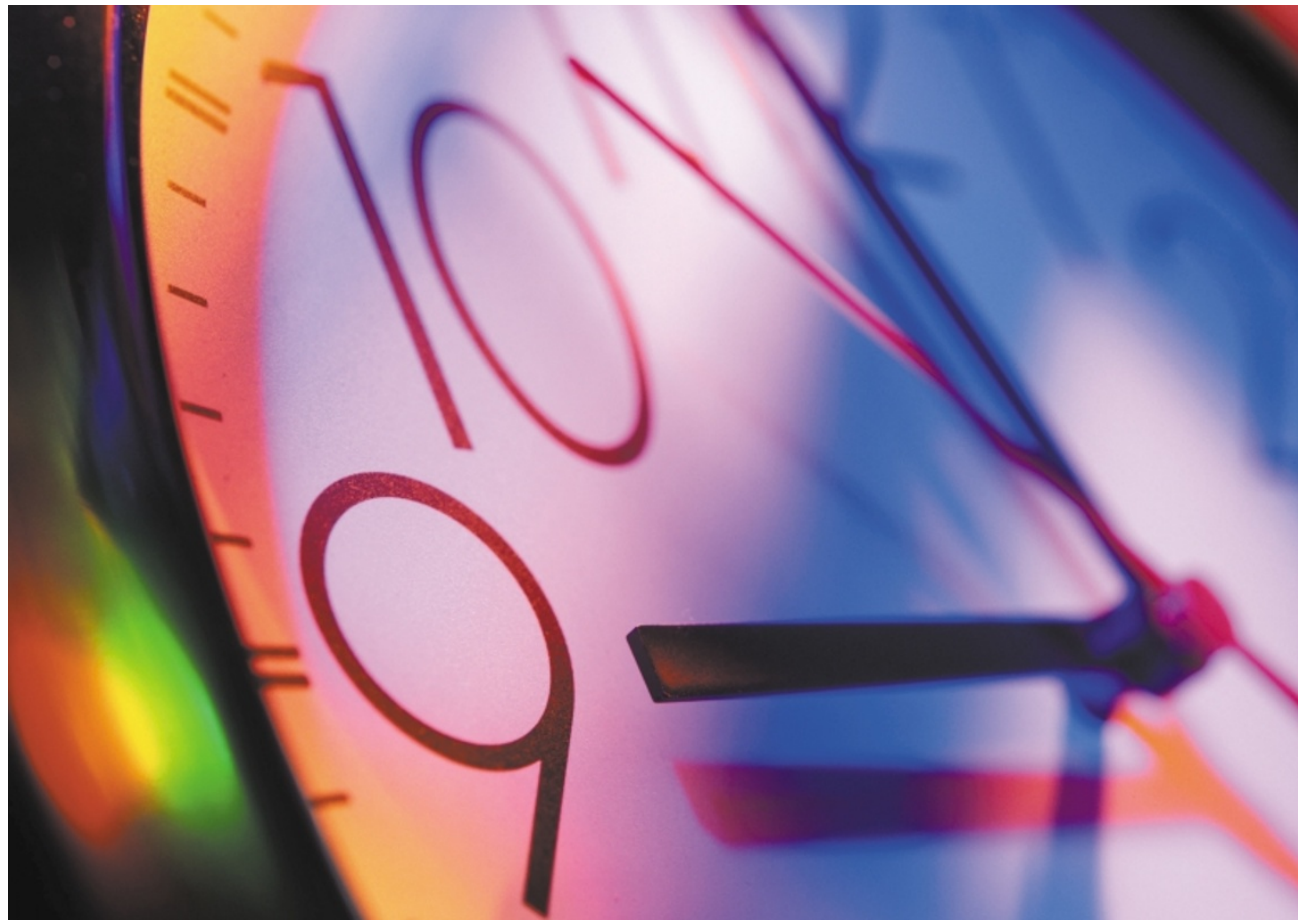

\section{BEHAVIOURAL GENETICS}

\section{Watching the clock}

The identification of the Drosophila period (per) locus - published in 1971 by Ronald Konopka and Seymour Benzer - was a singular landmark in behavioural genetics research. They were the first to show that genetics could be used to dissect behaviour, in this case the internal clock, or circadian rhythm, that underlies many aspects of behaviour in diverse organisms. Since then, many more genes involved in circadian rhythms have been identified (at least nine in mammals); the basic molecular mechanism of the clock has been defined; and the general features of the clock are known to be conserved in most living organisms. But little is known about the connections between the cellular workings of the clock and the patterns of behaviour that it influences. Two new studies provide valuable insights into how such knowledge might be obtained.

The study by Zheng et al. begins with an analysis of the mouse gene Per (mPer 1$)$ - one of three mammalian per homologues. By knocking out $P e r$, the authors show that its clock functions differ from those of Per2 ( $\mathrm{mPer} 2)$. This reinforces the idea that the core mechanism of the mammalian clock is more complex than in Drosophila. To investigate further the different roles of Per and Per2, the authors used microarray studies to identify genes that might be regulated by the clock in Per, Per2 and double-knockout mutants. They found 16 genes with reproducible circadian expression that was dependent on Per, Per2, or both genes. Two genes in particular showed circadian expression and were dependent on both Per and Per2: Alas1 and Alas2, which encode rate-limiting enzymes involved in haem biosynthesis. Because haem is an essential part of many proteins, such as metabolic and signalling proteins, the authors speculate that the circadian regulation of these genes might feed into a range of physiological processes.

In the second study, Shimomura et al. used a quantitative genetics approach to identify loci that underlie the difference in circadian rhythms (as measured by wheel-running behaviour) between two inbred mouse strains. Their phenotypic analysis measured five aspects of circadian behaviour, such as the length of the circadian cycle and the phase angle of entrainment - how the onset of activity relates to the light-dark cycles. In addition to searching for independent quantitative trait loci, the authors also looked for epistatic interactions between loci, by incorporating a genome-wide search for loci that had an effect only in combination with another locus. They found 14 loci that had a significant effect on circadian behaviour, including several that interacted epistatically. Importantly, most of these loci mapped to regions outside those containing known mammalian circadian genes.

In the 30 years since period was first identified, geneticists have built on the sound foundations laid by the pioneers of this field, but before the vision of Benzer and his colleagues can be realized, a good deal more work needs to be done. Both of these new lines of research hold great promise for finding new genes that function somewhere between the cellular clock and its output — circadian behaviour.

Mark Patterson

\section{(0) References and links}

ORIGINAL RESEARCH PAPERS Zheng, B. et al. Nonredundant roles of the $m P e r 1$ and $m P e r 2$ genes in the mammalian circadian clock. Cell 105, 683-694 (2001) | Shimomura, K. et al. Genome-wide epistatic interaction analysis reveals complex genetic determinants of circadian behavior in mice. Genome Res. 11, 959-980 (2001)

FURTHER READING Weiner, J. Time, Love and Memory (Faber \& Faber, New York, 1999)

WEB SITES Cheng Chi Lee's lab | Joe Takahashi's lab 